\title{
Functional Network Analysis Reveals Versatile MicroRNAs in Human Heart
}

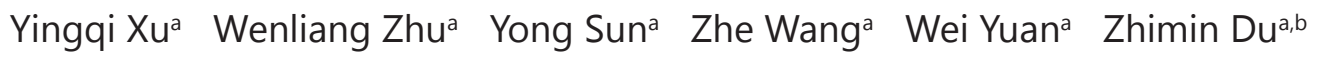 \\ Institute of Clinical Pharmacology, the Second Affiliated Hospital of Harbin Medical University, Harbin, \\ ${ }^{b}$ Department of Pharmacology (the State-Province Key Laboratories of Biomedicine-Pharmaceutics of \\ China, Key Laboratory of Cardiovascular Research, Ministry of Education), Harbin Medical University, \\ Harbin, China
}

\section{Key Words}

MicroRNA • Myocardial infarction $\cdot$ Heart failure $\cdot$ MicroRNA-7

\begin{abstract}
Background: Acting on many mRNAs allows the power of a single miRNA to modulate multiple pathophysiological phenotypes. One question is whether versatile miRNAs exist in the pathological scenarios of myocardial infarction (MI) and heart failure (HF). Methods: A hypergeometric analysis, in combination with network-based functional analyses, was performed on the available human protein interaction and miRNA-gene association data to highlight versatile miRNAs among the significantly dysregulated miRNAs in MI and HF. In vivo, mice models of MI and HF were then established to investigate whether dysregulated expression be undertaken by versatile miRNA identified here. Results: Systematic analyses really identified the previously validated miRNAs that have been verified of multiple important roles in $\mathrm{MI}$ and $\mathrm{HF}$, demonstrating method effectiveness. By using this means, we innovatively revealed the vital role of miR-7 in maintaining the dynamic balance of protein interactions and its obvious overexpression in MI and HF that implies pathological involvement. Functional experiments are definitely needed for further revealing its potential influences on MI- or HFled myocardial injury. Conclusion: Our results have implications not only for the coming miRNA-based strategy in treating MI and HF but also for further understanding on gene regulation by miRNAs in human heart.
\end{abstract}

Copyright (C) 2015 S. Karger AG, Basel

\section{Introduction}

MiroRNAs (miRNAs) play a central role in regulating some key protein-coding genes related to nearly all pathophysiological processes of human diseases [1]. With the important functions of miRNAs in cardiovascular disease continuously brought to light, the clinical applications of miRNAs have been prospectively and broadly attentioned [2-7]. Some studies 


\section{Cellular Physiology Cell Physiol Biochem 2015;36:1628-1643 \begin{tabular}{l|l} 
DOI: 10.1159/000430324 & $\begin{array}{l}\text { O 2015 S. Karger AG, Basel } \\
\text { www.karger.com/cpb }\end{array}$ \\
\hline
\end{tabular} \\ Xu et al.: Revealing Versatile MicroRNAs in Heart}

have shown that miR-122 can reduce the plasma cholesterol level [8]. Thus, it shows the feasibility of miRNA-based therapy to some human disease. However, a more important question remains: among the numerous of miRNAs, which miRNA should be the next and preferential target of human diseases. It is extremely difficult to screening comprehensive therapeutic target among miRNAs only using traditional biological experiments. Thus, armed with a large quantity of bioinformatics tools and resources, network-based systems biology has proved itself an alternative strategy to gain insight into this issue [9].

The complementary binding mechanism of miRNA-gene interaction [10] generates the innate diversity of miRNA regulation according to different base sequences [11]. This diversity may explain why overexpression of miR-195, but not miR-214, induced cardiac hypertrophy [12]. Pan et al. proposed that successful reversal of pathological processes can be obtained by solely modulating some key miRNAs [3]. But due to the complex pathogenesis of cardiovascular diseases, we thought that a suitably targeted miRNA should not be only marked as 'key', but defined as 'versatile'. The versatile miRNA could induce significant alteration of pathological phenotypes, because versatile miRNAs usually participate in multiple biological process and more pathological processes could be successfully reversed by restoring it to normal levels. MiRNAs exert function mainly through 'fine-tuning' their target gene expression. Therefore, we performed a hypergeometric analysis of functional protein network and miRNA-gene interaction data to prove the existence of the versatile miRNAs in human heart. Especially, as the miRNA expression profiles of human myocardial infarction (MI) and heart failure (HF) were available by far [13-15], we further explored among the significantly dysregulated miRNAs implicated in MI and HF. Each miRNA was underwent a comprehensive assessment. Static topological parameter calculation described its regulation capacity and tendency of targeting hub-encoding genes which is the central part of biological network (Fig. 1A). Dynamic perturbation simulation quantified the efficiency of miRNA regulation against the robustness of functional network. We identified the enriched biological processes that it might be preferentially involved in the context of GO (Fig. 1B). And moreover, the miRNA-disease association analysis would reveal whether it was significantly related to the disease phenotype of MI or HF (Fig. 1C).

$\mathrm{HF}$ is associated with significant morbidity and mortality, commonly caused by MI and trigger life-threatening arrhythmias and sudden cardiac death due to electrical conductance abnormalities [16]. On account of important roles in gene regulation, miRNAs may be indispensable involvers in their pathogenesis [13-15]. With the proposed network biology approach, designers are able to identify versatile miRNAs implicated in human MI and HF. It can contribute to the coming era of miRNA-based therapeutic strategy for patients with cardiovascular diseases. Intriguingly, in posttranscriptional regulation, miRNAs participate in many cellular pathways in both physiological and pathological conditions [1]. Therefore, undoubtedly, the cardiovascular disease paradigm of identifying versatile miRNAs using network biology approach is also applicable to other human diseases.

\section{Materials and Methods}

\section{Animals}

Healthy male KunMing mice (25-30 g) were maintained under the standard animal room conditions (temperature $21 \pm 1{ }^{\circ} \mathrm{C}$; humidity 55-60\%) with food and water ad libitum for one week before the in vivo experiments. The use of animals complied with the Guide for the Care and Use of Laboratory Animals published by the US National Institutes of Health (Eighth Edition, 2011) and pre-approved by the Experimental Animal Ethic Committee of the Harbin Medical University, China (Animal Experimental Ethical Inspection Protocol, No. 2009104).

Genomic and cardiac-specific protein interaction networks

Genomic protein interaction network (GPIN) was established by importing the protein-encoding gene symbols of HGNC [17] into the Cytoscape [18] plugin BisoGenet [19] with the BioRelation type option 


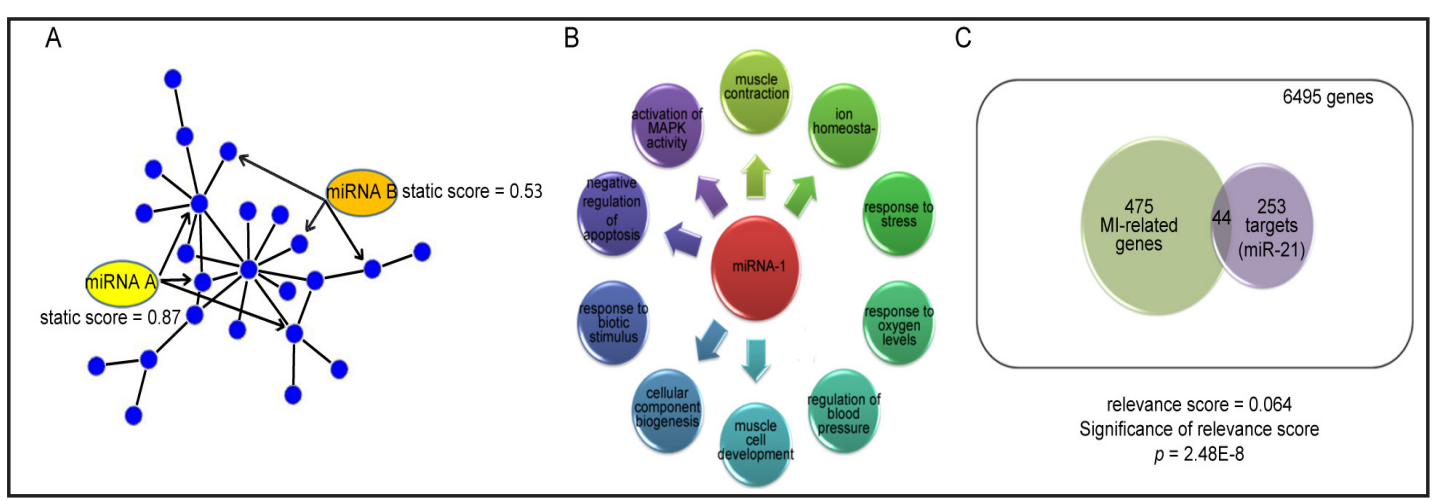

Fig. 1. Illustration of the multiple network analyses upon miRNAs. A. Schematic diagram of static score calculation. B. Result of enriched biological process identification with miR-1 as an example. The significantly down-regulated genes in ischemic cardiomyopathy were responsible for the 10 significantly over-presented biological processes. C. Result of miRNA-disease association analysis with miR-21 as an example (gene set, miRSel+TargetScanHuman).

'protein-protein interaction'selected. BisoGenet is a Cytoscape plugin that was designed for building biological networks through integrating data from different sources. GPIN was then processed by another plugin NetAtlas to identify cardiac-expressing genes encoding proteins in the network by filtering highthroughput tissue gene expression data in humans [20]. As the filter option 'Mean' was set, the gene expression data of human heart was chosen to create a sub-network. By combining with the NetAtlas array genes experimentally validated to be expressed in heart [21], the genes encoding proteins in the subnetwork were imported into BisoGenet again to create cardiac-specific protein interaction network (CPIN). Finally, the gene expression data of cardiac tissue was imported into CPIN as node attribute called 'Gene abundance'.

\section{Static topological parameter calculation}

To assess the regulatory capacity of each miRNA significantly dysregulated in HF and MI [13-15], the Cytoscape plugin NetworkAnalyzer [22] was firstly used to analyze the topological feature of its literaturereported [23] and predicted target genes [24-27] in three independent target gene sets (miRSel+MicroCosm, miRSel+PicTar, and miRSel+TargetScanHuman). NetworkAnalyzer is a built-in plugin for calculating network topological parameters in Cytoscape. Notably, if 2 gene sets of a miRNA were comprised of less than 100 genes, the miRNA would be discarded from our analysis. Two irrelevant topological parameters degree and neighborhood connectivity were selected to calculate the integrated parameter static score as defined in our previous study [28], which is used to evaluate the regulatory capacity of each miRNA. For each miRNA, static score was defined as the sum of the normalized mean degree and neighborhood connectivity values of its target genes subtracted by 3.3. We calculated three static scores for a miRNAs based upon different target gene sets in CPIN. The mean static score was then used to describe the overall topological status of proteins encoded by its target genes. Because CPIN was a truncated network from GPIN, we also repeated the static score calculation in GPIN for these miRNAs. Especially, we applied another target gene set (miRSel+miRecords). As prediction results are often vacant in miRecords for some integrated prediction programs, we only used 6 prediction programs (miRanda, MirTarget2, PicTar, PITA, RNAhybrid and TargetScan/TargertScanS) and defined that confidently trusted miRNA-gene interactions must be jointly predicted by at least 4 prediction programs [25]. Because highly connected proteins will definitely play more important roles in cellular functions than other proteins [29], we applied the classification scheme used by Camargo A and Azuaje F [30]. All nodes in GPIN were categorized into 4 categories [suerper-hub (degree $\geq 100)$, hub $(100>$ degree $\geq 20)$, Peripheral-A $(20>$ degree $\geq 2)$ and Peripheral-B(degree $=1)]$.

\section{Hypergeometric test}

We evaluate the significance of the dysregulated miRNAs targeting super-hub and hub encoding genes by using hypergeometric test. The hypergeometric probabilities was calculated as follows:

\section{KARGER}




\section{Cellular Physiology Cell Physiol Biochem 2015;36:1628-1643 \begin{tabular}{l|l}
\hline DOI: 10.1159/000430324 & (c) 2015 S. Karger AG, Basel
\end{tabular} and Biochemistry Published online: July 10, 2015

$$
P=1-\sum_{k=0}^{m} \frac{\left(\begin{array}{l}
n \\
k
\end{array}\right)\left(\begin{array}{l}
N-n \\
M-k
\end{array}\right)}{\left(\begin{array}{l}
N \\
M
\end{array}\right)}
$$

Where $\mathrm{N}$ is the total number of genes in the GPIN, $\mathrm{M}$ is the total number of super-hub and hub encoding genes in the GPIN, $n$ is the number of all of the target genes of a miRNA, $m$ is the number of super-hub and hub encoding genes targeted by the given miRNA. For comparing the significant tendency of these miRNAs in the whole genome, all miRecords predicted human miRNAs will undergo this assessment. Similarly, the miRNAs possessing less than 100 target genes encoding proteins in GPIN would be discarded from our analysis. To verify the result of hypergeometric probabilities in GPIN, we calculated the mean super-hub and hub count using the above mentioned three independent target gene sets for each dysregulated miRNA in CPIN. By targeting more super-hub and hub encoding genes, a miRNA is believed able to produce more vigorous phenotypic changes if it carries out significantly altered expression.

\section{Dynamic perturbation simulation}

To evaluate the regulatory efficiency of a miRNA against network robustness [31], dynamic score was then calculated as described in our previously study [28]. Firstly, the Cytoscape plugin PertubationAnalyzer was introduced to perform perturbation simulation with its target genes as perturbation sources and gene expression data as concentration values in CPIN [32]. Perturbation Analyzer is a Cytoscape plugin that assess perturbation-induced alternation in protein concentration by comparing before and after equilibrium states. The manual mode was selected. Then, dynamic score of a miRNA was calculated as the ratio of the property Perturbed subgroup size at 2.0 and 1.2 subgroup thresholds. As proteins encoded by the targeted genes of a miRNA were selected as perturbation sources, dynamic score was used to assess the global effect of altered miRNA expression on the homeostasis of CPIN. The higher dynamic score means more efficient regulation by the altered miRNA.

\section{Identification of enriched biological processes regulated by miRNAs}

Although the experimentally validated roles of miRNAs were taken into deliberate consideration upon their targetable potential, it had to be admitted that such biological evidence was still limited. So, to reveal the biological processes that a miRNA might be preferentially involved in, the DAVID functional annotation clustering tool [33] was performed upon the target genes with the entire list of genes in CPIN as background. 'GOTERM_BP_ALL' was chosen as the only functional annotation. The classification options 'Initial Group Membership' and 'Final Group Membership' were set 2. The threshold of Enrichment Score of 1.20 was used to identify the significantly over-presented biological process [33].

\section{MiRNA-disease association analysis}

The gene microarray data of dilated cardiomyopathy (DCM) and ischemic cardiomyopathy (ICM) was downloaded from the GEO database. There were totally five datasets for DCM and ICM, respectively (Table 1). We identified the differentially expressed genes that associated with DCM or ICM in each dataset, using the significance analysis of microarray (SAM) method (FDR $<0.05)$ and the Fold-change method (FC

Table 1. The analyzed gene microarray datasets of human heart failure. The analyzed gene microarray datasets of human heart failure. DCM: Dilated cardiomyopathy; ICM: Ischemic cardiomyopathy

\begin{tabular}{lccc}
\hline Accession number & $\begin{array}{c}\text { Sample of normal } \\
\text { heart (n) }\end{array}$ & $\begin{array}{c}\text { Sample of } \\
\text { DCM (n) }\end{array}$ & $\begin{array}{c}\text { Sample of } \\
\text { ICM (n) }\end{array}$ \\
\hline GSE1145 & 11 & 15 & 11 \\
GSE1869 & 6 & 21 & 10 \\
GSE3585 & 5 & 7 & 0 \\
GSE3586 & 15 & 13 & 0 \\
GSE5406 & 16 & 86 & 108 \\
GSE16499 & 15 & 0 & 14 \\
GSE17294 & 3 & 0 & 2 \\
\hline
\end{tabular}




\section{Cellular Physiology Cell Physiol Biochem 2015;36:1628-1643 \begin{tabular}{l|l|l}
\hline DOI: 10.1159/000430324 & C 2015 S. Karger AG, Basel
\end{tabular} \begin{tabular}{l|l} 
and Biochemistry Published online: July 10, 2015 & www.karger.com/cpb
\end{tabular} \\ Xu et al.: Revealing Versatile MicroRNAs in Heart}

$>1.5$ or FC < 0.66) simultaneously [34]. The significantly up- and down-regulated genes were respectively combined corresponding to the same disease-type. Notably, the gene names appearing in both the up- and down-regulated gene lists were deleted, so did the genes which transcript products could not be found in CPIN. To identify the MI-related genes, the Gene Prospector tool in the HuGE Navigator database was applied to explore gene evidence of ischemia [35]. Similar to miRNA targets, the possibly involved biological processes were also explored for the disease genes in DCM, ICM and MI using the DAVID tool. Besides of the introduced hypergeometric probability for evaluating the significance of miRNA-disease association $(p<0.05)$, a relevance score was calculated to quantify the relationship between a miRNA and disease in common gene involved as followed:

$$
\text { Relevance_score }=\frac{N_{\text {common__enes }}}{N_{\text {miRN__targets }}+N_{\text {disease_genes }}-N_{\text {common__enes }}}
$$

To re-validate the result of the analysis on miRNA-disease association, the online tool g:Profiler [36] was applied to perform GO analysis on the experimentally-validated [37] and literature-reported [23] target genes of each of the 51 dysregulated miRNAs in MI and the highly-confident MI-related genes [38]. In the website of g:Profiler, the minimum and maximum sizes of functional category was set as 5 and 50, respectively. Only the GO option biological process was selected for enrichment analysis. A BenjaminiHochberg FDR of $1 \times 10^{-4}$ was selected as significance threshold.

\section{Mouse models of MI}

Mice were anesthetized with ketamine-xylazine (100 mg/kg, $5 \mathrm{mg} / \mathrm{kg}$, i.p.), the mice was sufficiently anesthetized and nonresponsive to foot pinch, and intubated orally with a polyethylene tube (UGO Bsile S.R.L. Biological Research Apparatus, Italy) for artificial respiration. A left-sided thoracotomy was performed through the fourth intercostal space, and the pericardium was opened to expose the heart, the left descending coronary artery was ligated using an 8/0 silk thread to create infarction. Myocardial infarction was confirmed by apparent S-T segment elevation in ECG and cyanosis of the myocardium. The sham procedure was performed with a superficial suture in the epicardium of LV. After surgery the chest was closed and the animals were allowed to recover with food and water ad libitum. After 3 days, mice were killed with overdose sodium phenobarbital and the hearts were harvested for use.

\section{Transverse aortic constriction (TAC)}

After having been successfully anesthetized, the mouse was orally intubated with a 20-gauge tube and ventilated (UGO BASILE, Biological Research Apparatus, Italy). The transverse aorta was isolated gently and a 7-0 silk suture was snared with a 27-gauge blunt needle and pulled back around the aorta. Then, the blunt needle and the aorta were tied snugly around by the suture. After ligation, the needle was quickly removed to yield a constriction of $0.4 \mathrm{~mm}$ in diameter. Sham operated mice received identical surgical procedure except for ligation of the aorta, The chest and skin were closed and the pneumothorax was evacuated. Finally, the mice were allowed to recover from anesthesia. At 8 weeks later, mice were killed with overdose sodium phenobarbital, the hearts and lungs and the left ventricles were separated and weighed to calculate the heart weight/body weight (HW/BW, mg/g), left ventricular weight/body weight (LVW/BW, mg/g), lung weight/body weight (LW/BW, mg/g) ratios.

\section{Real-time PCR analysis}

Total RNA samples were isolated from mouse ventricular tissues using Trizol Reagent (invitrogen). The levels of miR-7 were determined using real-time RT-PCR method. One microgram of total RNA from each sample was used to generate cDNA by using M-MLV reverse transcriptase per manufacturer's specifications (Promega, USA). Real-time PCR was cycled at $95^{\circ} \mathrm{C} / 15 \mathrm{~s}, 60^{\circ} \mathrm{C} / 30 \mathrm{~s}$ and $72{ }^{\circ} \mathrm{C} / 30 \mathrm{~s}$ for 40 cycles, after an initial denaturation step at $95^{\circ} \mathrm{C}$ for $10 \mathrm{~min}$, using SYBR Green PCR Master Mix (Applied Biosystems, USA). Amplification was performed by using the 7500 Fast Real-Time PCR System (Applied Biosystems, USA). Relative quantitative method was used by normalizing the amount of detected transcripts to the internal control U6. The relative value to the control sample was given by $2^{-\Delta \Delta c t}$.

\section{KARGER}


Statistic analysis

Data were expressed as means \pm SEM. Comparisons among groups were tested by one-way ANOVA. Differences between two groups were determined by Student's $t$-test. Correlation between dynamic scores and static scores or mean gene abundance $(\log 2)$ was determined by Pearson test. A value of $p<0.05$ was considered to be statistically significant.

\section{Results}

High static scores and low hypergeometric probabilities revealed miR-1 and miR-21 in enriched regulation of vital genes

Understandably, the biological importance of proteins encoded by target genes of a miRNA ultimately determines its power in gene regulation. As the biological importance of a protein can be essentially reflected and measured by its topological status in functional protein network [29], we created a genomic protein interaction network [GPIN, including 119 super-hubs (degree $\geq 100)$ and 1522 hubs $(100>$ degree $\geq 20)$ contained] and evaluated the significant tendency of the 439 known human miRNAs in targeting super-hub or hub encoding genes by using hypergeometric test (See Materials and Methods). These miRNAs were retrived from the miRNA-target interaction databases including miRSel, MicroCosm, PicTar, TargetScanHuman and were found to target more than $100 \mathrm{mRNA}$ genes. The classification of network nodes on degree followed the scheme proposed by Camargo A and Azuaje F [30]. Twenty four miRNAs were distinguished by their assigned hypergeometric probability values $\left(p<1 \times 10^{-5}\right)$ and among them 16 underwent significantly altered expression in MI or HF (Fig. 2). Five miRNAs (miR-15a, miR-16, miR-107, miR-195 and miR424 ) belonged to the miR-15/107 group of miRNAs which key functions in human was only

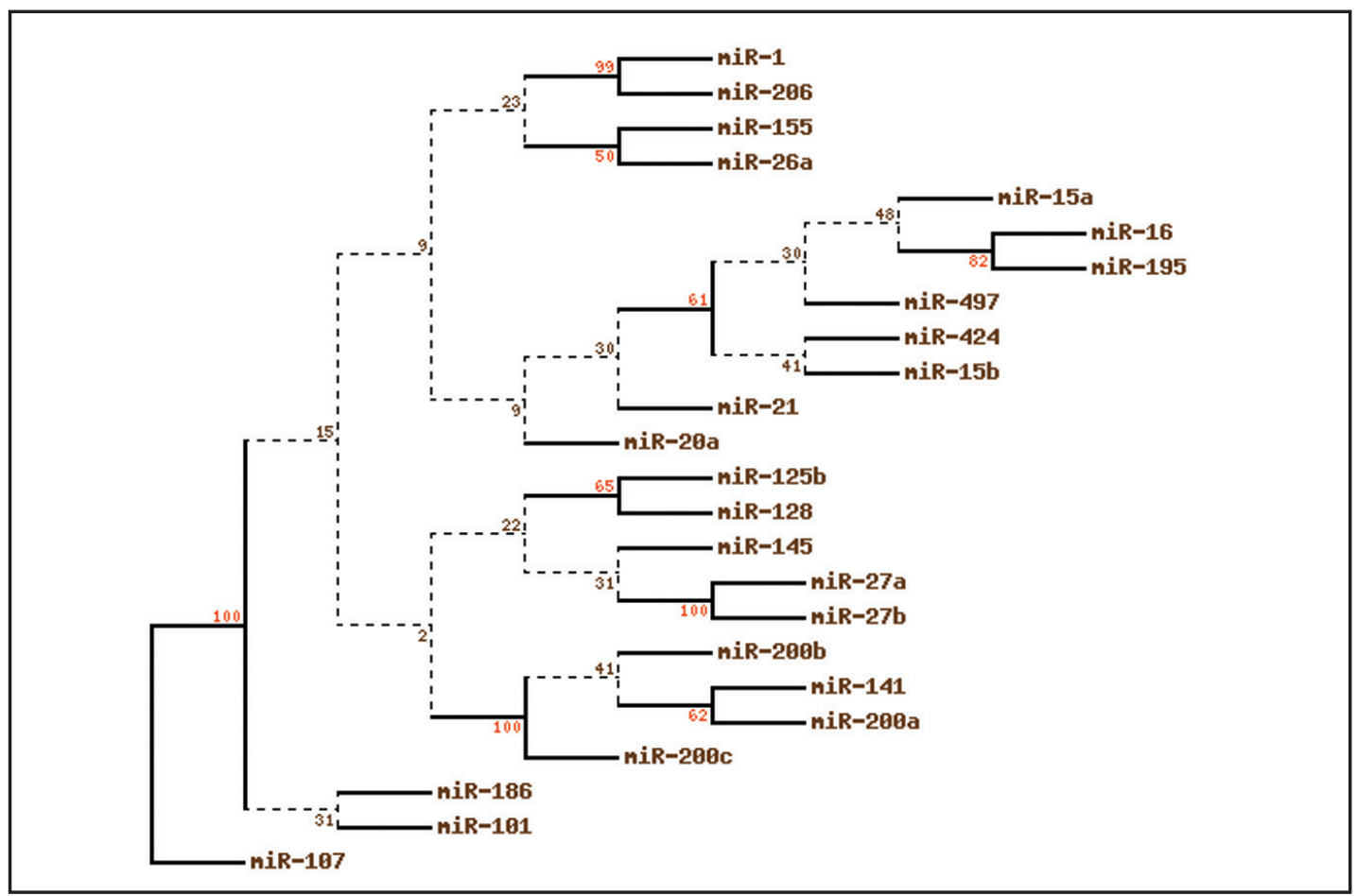

Fig. 2. The 16 dysregulated miRNAs significantly targeting super-hub and hub encoding genes. The graphic illustration of miRNA sequence similarity were generated using the online alignment tool GeneBee with the "Rectangular 2" cluster algorithm applied (http://www.genebee.msu.su/services/phtree_reduced.html). The bootstrap values were shown at nodes. 


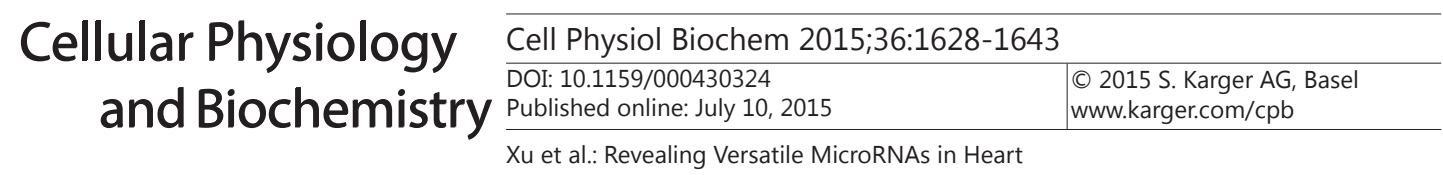

Table 2. Distribution of degree in CPIN and summary of neighborhood connectivity for each degree category. Distribution of degree in CPIN and summary of neighborhood connectivity for each degree category. CPIN: cardiac-specific protein interaction network; $N_{\text {node }}$ : the number of nodes; NC: neighborhood connectivity; SD: standard deviation. There were no statistical significant difference between category NC means at $p=0.05$. And the significant correlation between degree and $\mathrm{NC}$ was not found $(p=0.13)$

\begin{tabular}{lll}
\hline Category & $N_{\text {node }}$ & Average NC \pm SD \\
\hline Super-hub (degree $\geq 100)$ & 33 & $38.19 \pm 55.68$ \\
Hub $(100>$ degree $\geq 20)$ & 619 & $40.03 \pm 35.18$ \\
Peripheral-A $(20>$ degree $\geq 1)$ & 4419 & $37.36 \pm 15.73$ \\
Peripheral-B $($ degree $=1)$ & 1424 & $30.45 \pm 6.27$ \\
\hline
\end{tabular}

A

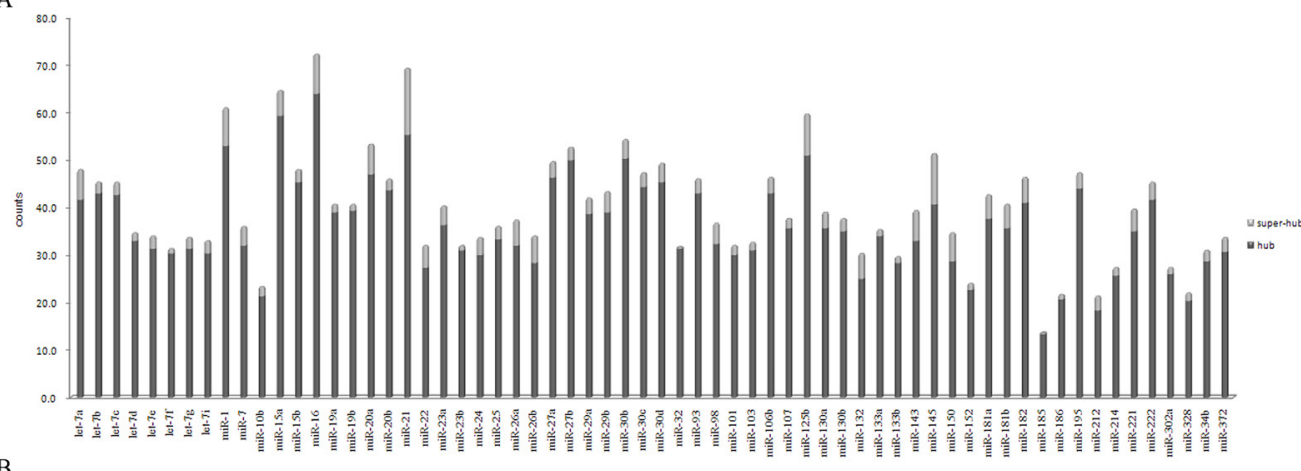

B

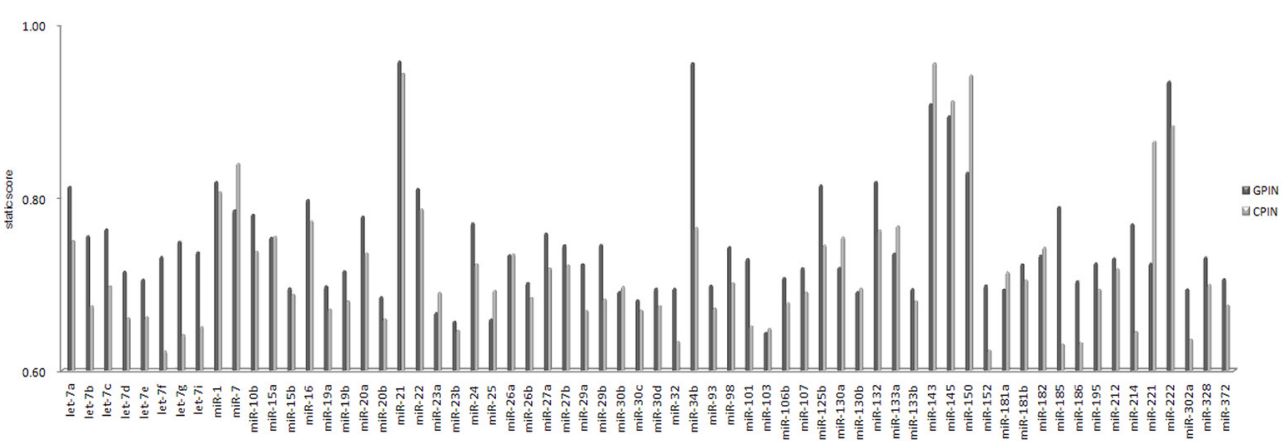

Fig. 3. Results of Static topological parameter calculation. A. Counts of the sum of super-hub and hub encoding genes targeted by each dysregulated miNRA. B. The dynamic scores of the 64 significantly dysregulated miRNAs in MI and HF. GPIN: Genomic protein interaction network; CPIN: cardiac-specific protein interaction network; MI: myocardial infarction; HF: heart failure.

recently well acknowledged [39]. Hypergeometric probability value was calculated for each miRNA included herein for enriched regulation on super-hub and hub encoding genes. MiR21 was assigned with the lowest probability value, implying its broad and important roles [40].

To evaluate the regulatory capacity of the significantly dysregulated miRNAs in MI and HF, we established a cardiac-specific protein interaction network (CPIN), in which 33 super-hubs and 619 hubs were found. Besides that the tissue-specificity was guaranteed 
Fig. 4. Results of dynamic perturbation simulation. A. Correlation between static score and dynamic score. B. Correlation between average target gene abundance (log2) and dynamic score. All significantly dysregulated miRNAs in MI and HF were analyzed, $(n=64)$.

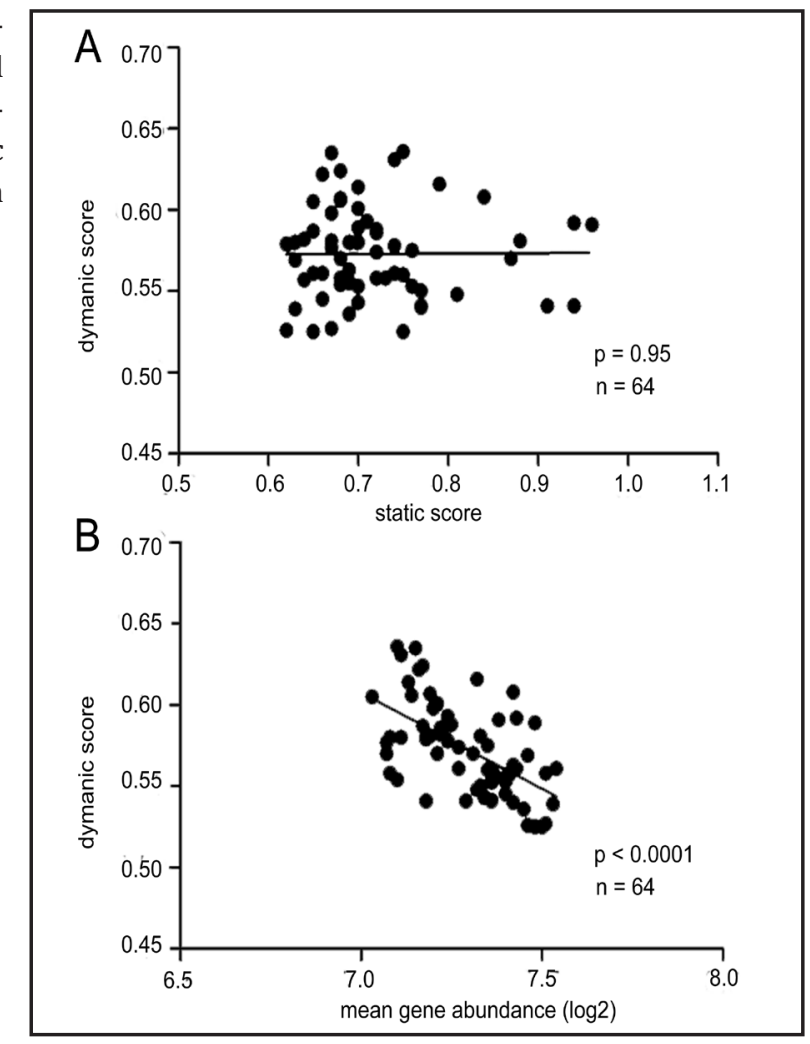

by the application of the Cytoscape plugin NetAtlas [20], more than 2700 nodes in CPIN represented proteins encoded by the validated genes expressed in human heart [21]. NetAtlas is a Cytoscape plugin that can be applied to identify tissue-defined network by using tissue gene expression data. After describing the topological structure of the network with two parameters degree and neighborhood connectivity (Table 2), we counted the sum of super-hub and hub encoding genes for each of the 64 miRNAs with significantly altered expression in MI or HF (Fig. 3A). As verified in GPIN (Fig. 2), the four miRNAs (miR-1, miR15a, miR-16 and miR-21) also regulated a large number of genes encoding super-hub or hub in CPIN $(\mathrm{n}>60)$. Then, we calculated the static score of these miRNAs (See Materials and Methods), which assess the regulatory capacity of miRNA significantly dysregulated in $\mathrm{HF}$ and MI. The result showed that only miR-1 and miR-21 were assigned with a high static score (static score $>0.80$ ) in both networks (Fig. 3B), implying the competitive biological importance of their targeted genes in whole.

Dynamic perturbation simulated the effect of miRNA-mediated gene regulation on network robustness

In this study, network robustness is defined as the resistance of biological protein-protein interaction network against external and internal disturbance. The fine-tuning of protein output by miRNA [41] made biological system robust to perturbation by environmental flux. Contrarily, this robustness could also counteract the impact exerted by miRNA and make its regulation less efficient. We calculated the dynamic score of each miRNAs (see Materials and Methods), which can evaluate the regulatory efficiency of a miRNA against network robustness. Dynamic score indicates the effects of perturbations in protein concentration of miRNA target genes in a protein interaction network and gain insight into the regulatory effect of miRNAs on dymamic and function of network. It was found that, instead of static score that described the connectivity characteristics of the network nodes, the dilution effect of target gene abundance [42] was partly responsible for the comparatively low dynamic scores of majority of miRNAs (Fig. 4A and 4B). Ten miRNAs obtained dynamic scores of more than 0.6, implying that altered expression of these miRNAs caused about two-thirds 
Fig. 5. Expression of miR-7 was significantly upregulated in the mice models of MI and HF. A. Reprehensive electrocardiograms of sham and MI mice. Comparison between TAC mice and Sham mice in HW/BW (B), LVW/BW (C) and LW/BW (D). *** $p<0.001$, TAC versus Sham; $\mathrm{n}=7$. Significant increase of miR-7 expression was observed in both the mice models of MI (E) and HF (F). * $p<0.05$, MI versus Sham; ${ }^{* * *} p<0.001$, TAC versus Sham; $n=4$.

A Sham

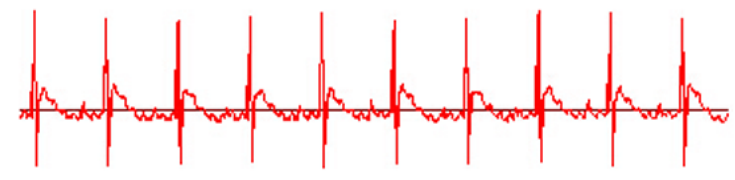

MI

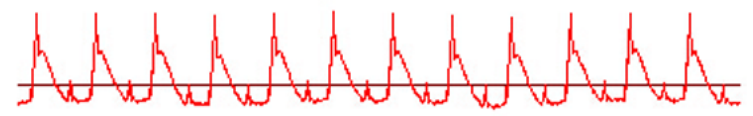

$\mathrm{B}$
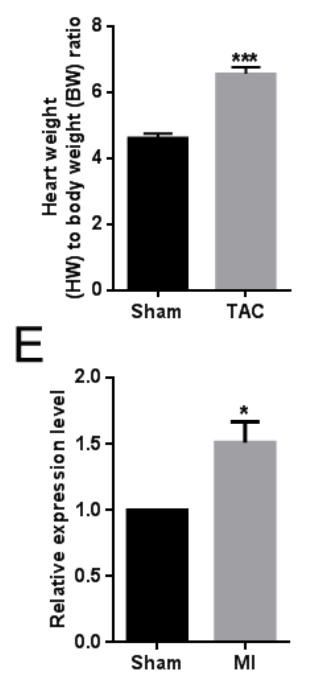

C

D
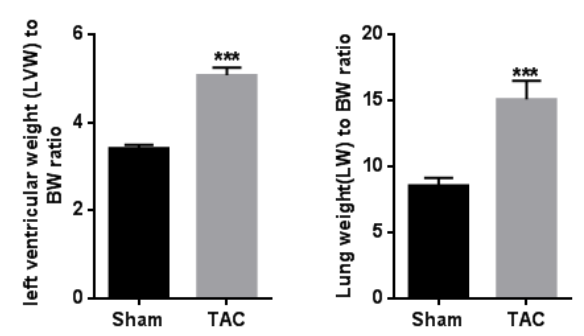

$\mathrm{F}$

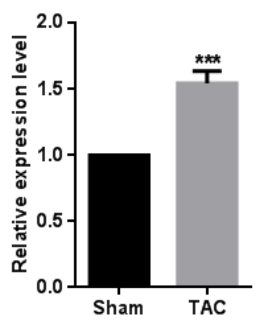

of all the perturbed proteins take a more than 2.0 ratio change in free protein concentration [32]. MiR-7 possessed the most competitive regulatory capacity (static score: 0.84 in CPIN). Comparatively, high static and dynamic scores made it the most sensitive regulator in all of the analyzed miRNAs. By targeting epidermal growth factor receptor and exerting influence on Akt signaling pathway [43,44], miR-7, an essential stabilizer of network regulation [45], confirmed its biological importance. Furthermore, we successfully established the in vivo animal models of MI and HF in mice (Fig. 5A-D). Real time-polymerase chain reaction (RT-PCR) assay then validated that miR-7 was significantly up-regulated in MI and HF ( $p<$ 0.05 , Fig. 5E and 5F). This finding implies real involvement of dysregulation of miR-7 in the pathologies of MI and HF.

A miRNA was prone to perform enriched regulation on genes involved in functionally associated biological processes

To investigate the functional roles of the 64 miRNAs altered in MI or HF, gene ontology enrichment analysis was performed based on their target gene sets. Based upon the identification results from two of the three target gene sets, these 64 miRNAs altered in MI or HF mainly participated in 8 dysregulated biological processes contributing to the pathophysiology of MI or HF (Fig. 6), such as tissue remodeling, apoptosis, protein stability, and signal transduction. Since participating in various biological processes, a miRNA might be assigned to several categories. For example, as regulating transcription factor activity and tissue regeneration miR-1 appeared in the two categories, transcription and 
Fig. 6. Results of enriched biological process identification. The similarity score of the number of miRNAs between different biological processes was calculated by referring to the formula of expression similarity (See Materials and Methods). Similarity scores of more than 0.2 were shown.

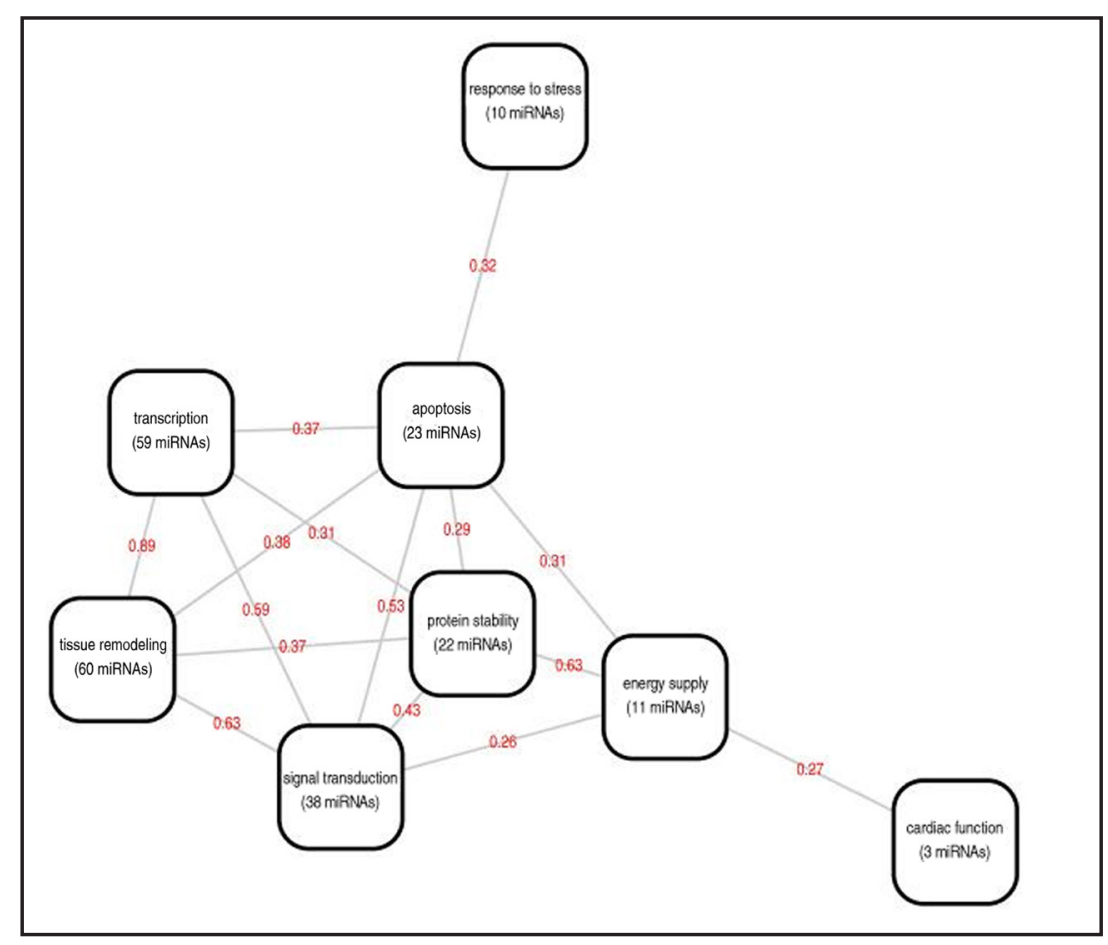

Table 3. The significantly over-presented biological processes in MI and HF. The significantly over-presented biological processes in MI and HF. MI: myocardial infarction; HF: heart failure; DCM: Dilated cardiomyopathy; ICM: Ischaemic cardiomyopathy; ES: Enrichment Score; the superscript a indicates that the relevance score of miR- 1 is more than 0.4 to the disease; the superscript $b$ indicates that the relevance score of miR- 21 is more than 0.4 to the disease; the superscript $c$ indicates MI-related genes retrieved from the HuGE Navigator database with the Gene Prospector tool applied to explore gene evidence of ischemia

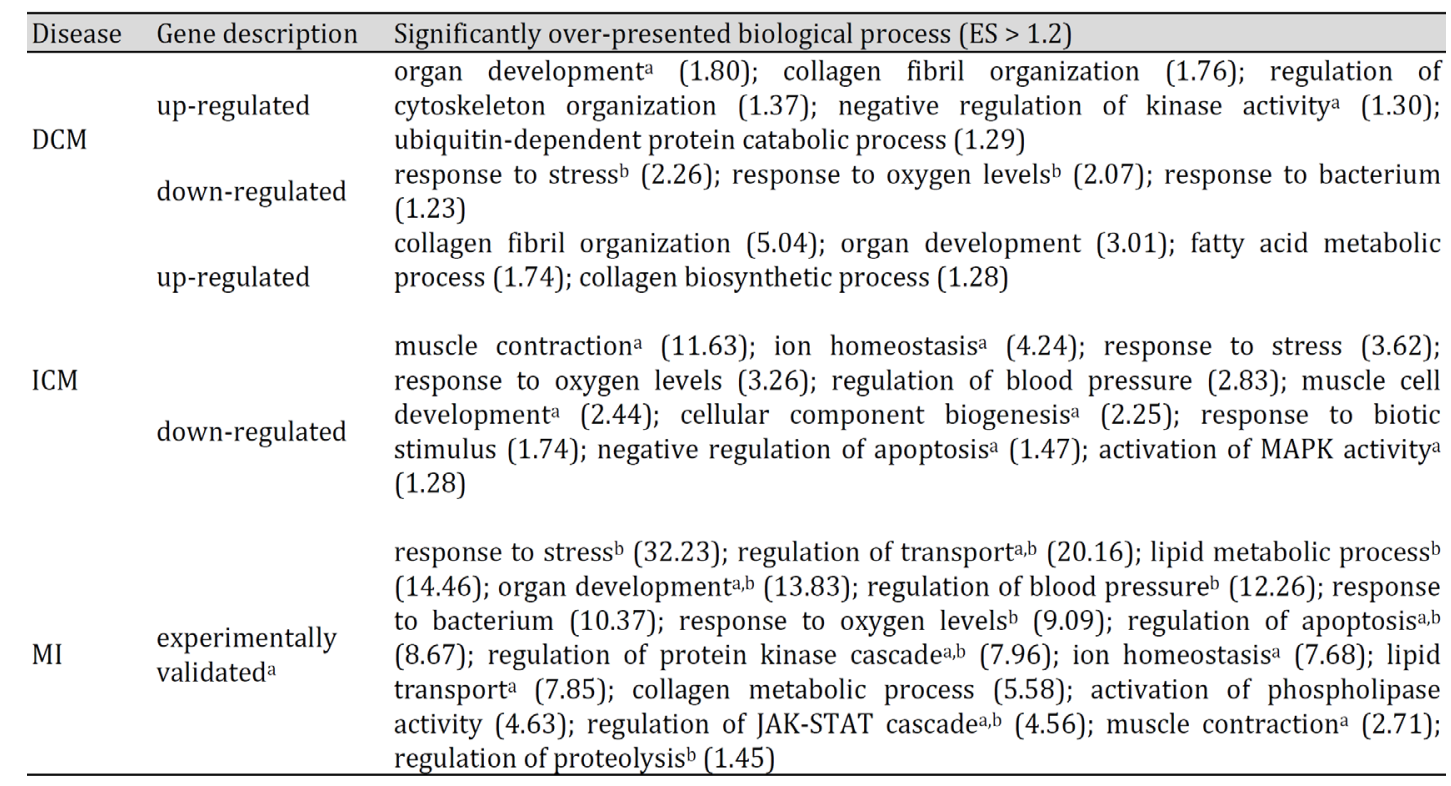

tissue remodeling. As shown in Fig. 6, most miRNAs put their regulatory emphasis upon tissue remodeling (60 miRNAs) rather than cardiac functions (3 miRNAs). In the light of the potentially important roles of miRNAs in cardiac remodeling [46], it was suggested that therapeutic strategies aimed at preventing or reversing pathological remodeling of the failing heart [47] might be more rational and effective than simply repairing cardiac dysfunctions 


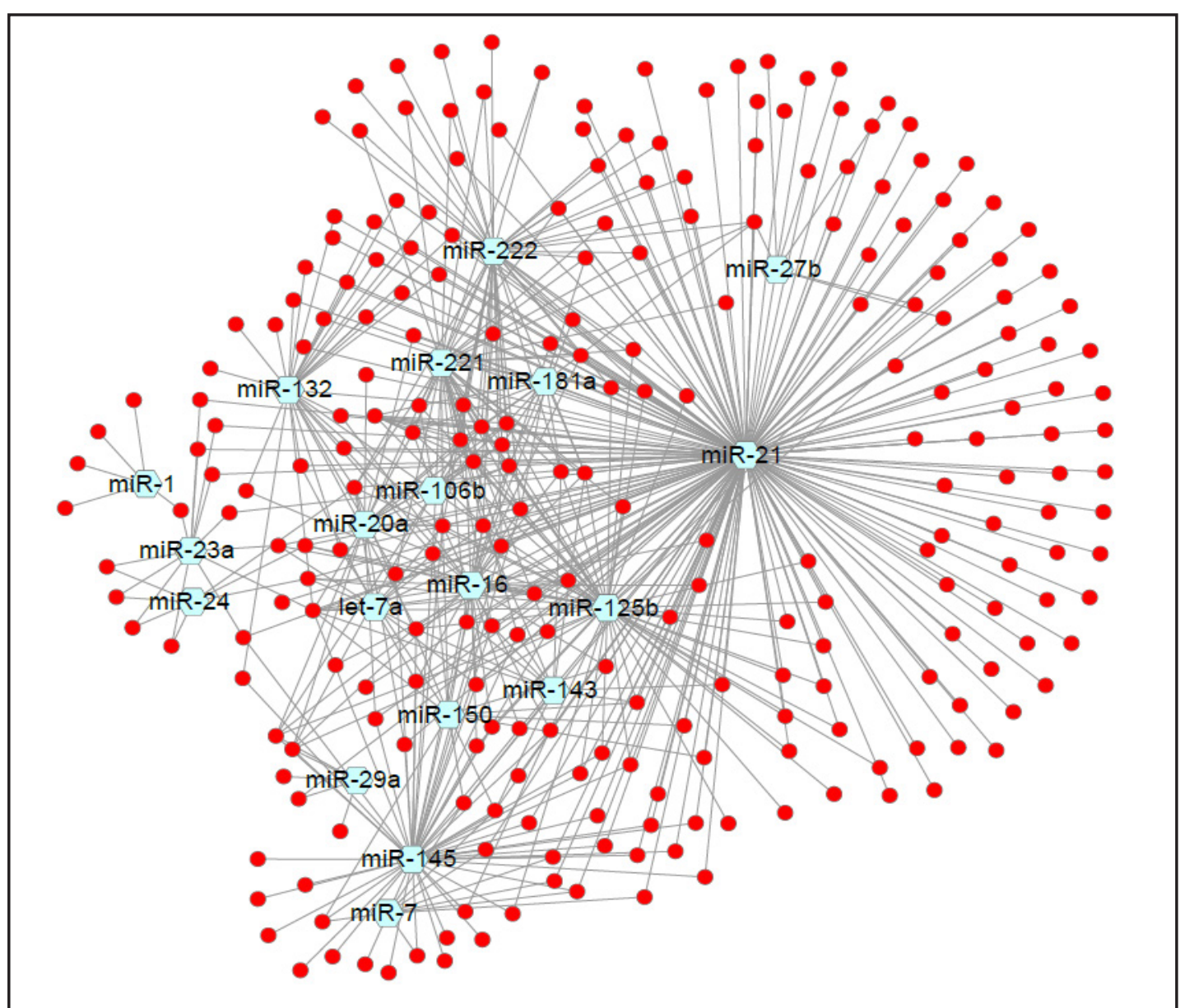

Fig. 7. GO analysis using g:Profiler re-verified involvement of miR-1 and miR-21 in regulating multiple MI-related biological processes. Red nodes represent MI-related biological processes. Edges mean significant overrepresentation of target genes of a miRNA on the biological process (FDR $<1 \times 10^{-4}$ ). Only the miRNAs regulating > 3 MI-related biological processes were shown.

such as antiarrhythmic therapy by using ion channel blockers [48]. We further revealed that the intrinsic link existed between the biological processes regulated by these 64 miRNAs and found that functions regulated by these miRNAs were closely connected (Fig. 6). For example, a miRNA regulating cardiac functions such as muscle contraction or ion homeostasis was very likely to modulate energy supply such as fatty acid oxidation. A miRNA participating in cellular response to oxidative stress might also contribute to cellular apoptosis as these two biological processes were functionally associated (Fig. 6).

After the SAM analysis of the dilated cardiomyopathy (DCM) and ischemic cardiomyopathy (ICM) gene microarray data retrieved from GEO (Table 1), we obtained 1397 significantly dysregulated genes encoding CPIN-contained proteins in DCM (916 genes up-regulated and 481 down-regulated) and 1078 in ICM (556 genes up-regulated and 522 down-regulated). By applying the Gene Prospector tool in the HuGE Navigator database [35], 475 validated MI-related genes were found, which encoded proteins in CPIN. The DAVID functional annotation clustering tool [33] identified the over-presented biological processes they were involved in (Table 3). Notably, three biological processes including collagen biosynthesis, protein kinase activity and response to oxygen levels were significantly changed in DCM, ICM and MI. Increased collagen biosynthesis implied the occurrence of myocardial fibrosis; altered protein kinase activity indicated the possibly ongoing cardiac remodeling; damaged response to oxygen levels increased the susceptibility of myocytes to various injuries. 


\section{Cellular Physiology Cell Physiol Biochem 2015;36:1628-1643

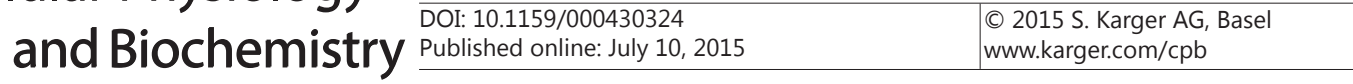 \\ Xu et al.: Revealing Versatile MicroRNAs in Heart}

MiRNA-disease association analysis revealed remarkable involvement of miR-1 and miR21 in $M I$ and $H F$

Although the miRNAs analyzed here was found significantly changed in human MI or HF [13-15], we still evaluated the statistical significance of the association between these miRNAs and MI or HF, with the introduced relevance score to quantity the miRNA-disease association. Relevance score assessed the potential functional association between a miRNA and disease by calculating the ratio of the common genes shared by miRNA target set and disease related gene set (See Materials and Methods). Among the 25 miRNAs correlated with the up-regulated genes in DCM (relevance score $>0.04, p<0.05$ ), miR-1 and miR-145 possessed higher regulatory capacity (static score $>0.8$ ), significantly regulated superhub and hub-encoding genes (Fig. 2 and $3 \mathrm{~A}$ ) and participated in $\geq 2$ enriched biological processes of DCM. Comparatively, only miR-21 passed such strict screening among the 13 miRNAs that was significantly associated with the down-regulated genes in DCM. There were 19 miRNAs with relevance score of more than 0.04 to the up-regulated genes in ICM. Except miR-29a/b which contributed to the development of cardiac fibrosis, no miRNAs with higher regulatory capacity (static score $>0.8$ ) and multiple regulation $(\geq 2$ enriched biological processes) were found. Although both miR-1 and miR-16 were significantly associated with the down-regulated genes in ICM (relevance score $>0.04, p<0.05$ ), higher regulatory capacity and involvement in 6 enriched biological processes including muscle contraction and ion homeostasis made miR-1 more competent as therapeutic target for ICM. In a similar screening focused upon the dysregulated miRNAs in MI, miR-1 (relevance score: 0.043 and 8 enriched biological processes) and miR-21 (relevance score: 0.064 and 10 enriched biological processes) demonstrated their therapeutic potential for ischemic heart disease (Table 3). Additionally, after integrating the experimentally-validated [37] and the literature-reported [23] evidence of miRNA-target interactions, an alternative GO analysis was performed by using the online tool g:Profiler [36]. Significant overrepresentation of 710 GO biological processes was also identified for the highly-confident 604 MI-related genes [38] (false discovery rate, FDR $<1 \times 10^{-4}$ ). Consistent with the result of DAVID analysis, the present finding re-verified that miR-1 and miR-21 significantly regulate multiple MI-related biological processes (Fig. 7). Especially, compared with extensive gene regulation executed by miR-21, miR-1 was observed to be specially enriched in regulation of cardiac muscle contraction and heart growth.

\section{Discussion}

Up to now, it is estimated that more than 1000 miRNAs are encoded by human genome. Each of them acting on tens to hundreds of genes to modulate complex physiological or disease phenotypes [1] Van Rooij et al. reported that miR-214 together with other four miRNAs were significantly up-regulated during cardiac hypertrophy. However, the authors found that only over-expression miR-214 could not induce cardiac remodeling. This suggested that only significant changes in expression level could not definitely mean that a miRNA would play an important role [12]. In the present study, we performed a series of network-based assessments (Fig. 1) upon the miRNAs that significant altered expression in human MI or HF [13-15] to find the potential versatile miRNAs.

Among the analyzed miRNAs, which were implicated in MI or HF. Four miRNAs (miR-1, miR-15a, miR-16 and miR-21) showed their prominent tendency in targeting genes encoding super-hubs and hubs (Fig. 2 and 3A). Especially, for miR-21, its significant preference was still competitive in the context of the entire genome, implying its broad and important roles [40]. The enriched appearance of the miR-15/107 group of miRNAs in Fig. 2 was consistent with their received increasing appreciation for serving pivotal biological roles [39]. In addition, static score were used to quantitatively evaluated the overall topological statuses of the targeted genes for each miRNA. Network Analyzer [38] was responsible for calculating static score, an integrated result of 2 topological parameters (See Materials 


\section{Cellular Physiology Cell Physiol Biochem 2015;36:1628-1643 \begin{tabular}{ll|l} 
and Biochemistry Published online: July 10, 2015 & $\begin{array}{l}\text { DO 2015 S. Karger AG, Basel } \\
\text { www.karger.com/cpb }\end{array}$ \\
\hline
\end{tabular} \\ Xu et al.: Revealing Versatile MicroRNAs in Heart}

and Methods). Presumably, a comprehensive description of node in functional network can be provided by using more topological parameters. Totally 9 topological parameters were initially considered into static score calculation for each miRNA. Because of the existed correlation between parameters, only degree and neighborhood connectivity were remained. Degree counts the number of neighbors connected to a node. Highly connected nodes as defined as the hubs will definitely play more important roles in cellular functions than other proteins [2]. It is very likely that a protein with high neighborhood connectivity also join in important cellular functions due to the likelihood of the connection with a hub [49]. Available biological evidence confirmed a strong regulation of miR-1 and miR-21 that were highlighted with comparatively higher static scores (Fig. 3B). There are increasing lines of evidence suggesting that miR-1 might be a new potential therapeutic target for MI [50]. Further, Lu et al. confirmed the anti-ischemic cardioprotection provided by inhibition of miR-1 [51]. Taken together, these studies provide the functional links between miR-1 and MI. Increasing evidence demonstrated that miR-1 was also involved in cardiac hypertrophy, a leading cause of heart failure, and overexpression of miR-1 inhibited hypertrophic growth of cardiomyocytes [52]. Being similar to the prominent roles of miR-1 in the cardiovascular system, the therapeutic potential of miR-21 has also been shown to HF by focusing upon the development of cardiac fibrosis [53].

Following the model introduced by Maslov [31], Perturbation Analyzer can assess the perturbation effect of protein concentration change by comparing before and after equilibrium states [32]. In this study, we used it to evaluate the regulatory efficiency of miRNAs against network robustness with dynamic score introduced (See Materials and Methods). Notably, because the genome-wide protein abundance data is not yet available [32], the mRNA abundance was used as an approximation of protein abundance to calculate the dynamic score (See Materials and Methods). This replacement was thought rational and acceptable [54]. Furthermore, it was also found that after miR-1 transfection to HeLa cells the mRNA levels were remarkably correlated with protein output [55]. Based upon the fact of the dilution effect of target gene abundance to miRNA regulation [41] and the good correlation between dynamic score and target gene abundance $(p<0.0001)$, we identified miR-7 as the most sensitive regulator. The important identity as an essential stabilizer of network regulation [45] and exerted influence on Akt signaling pathway [44] confirmed our conclusion. Remarkable dysregulation of miR-7 was validated on both the mice models of MI and HF (Fig. 5). However, more experimental evidences are definitely needed about aberrant expression of miR-7 induced possible deterioration of myocyte homeostasis.

The biological tissue remodeling was concentrated with more miRNAs implying that miRNAs might exert their impacts on it to alter the cardiac function (Fig. 6). This distinguishing feature of miRNAs emerged as a novel therapeutic strategy for cardiac remodeling [46]. In the enriched biological process identification of the dysregulated genes in MI and HF, continued cardiac remodeling during MI and HF could be also well reflected (Table 3). Furthermore, the inherent link between the biological processes regulated by miRNAs also revealed the possible radiation of miRNA regulation. If the operation of miRNAs in the regulation of energy supply module became abnormal, cardiac function and protein stability were impacted (Fig. 6). This chain reaction aroused by altered miRNA expression might imply that the suitable therapeutic target of miRNA should have more 'skills'. In the miRNA-disease analysis part, we further evaluated the statistic significance of the relationship between the dysregulated miRNAs and MI or HF. By setting up strict screening limits, miR-1 and miR-21 stood out from the miRNAs that altered expression in MI and HF. In our result, the versatile miRNAs that prominent tendency in targeting genes encoding super-hubs and hubs, higher static scores, significant association with MI and HF and involvement in more disease-related biological processes were the most appropriate therapeutic targets compared to other miRNAs. Additionally, the important role of miR-145 in DCM was also highlighted. It would receive more attention according to its cardiac-specific expression pattern and important identity in deciding mytocyte fate [56]. 


\section{Cellular Physiology Cell Physiol Biochem 2015;36:1628-1643 \begin{tabular}{ll|l} 
and Biochemistry 10.1159/000430324 & $\begin{array}{l}\text { Do 2015 S. Karger AG, Basel } \\
\text { Published online: July 10, } 2015\end{array}$ & \begin{tabular}{l} 
www.karger.com/cpb \\
\cline { 2 - 3 } and
\end{tabular} \\
\cline { 2 - 3 }
\end{tabular} \\ Xu et al.: Revealing Versatile MicroRNAs in Heart}

Although the core therapeutic strategies in HF were largely remained in the recent years [57], an increasing number of evidence has strongly suggested that the novel miRNAbased therapy represents a hopeful and imperative trend in the future [3]. However, the endless extending list of miRNAs on the miRBase website makes it difficult for target screening. Despite experimental evidence cannot be replaced, bioinformatics approach also has potential to provide insights into the pathophysiological involvement of miRNAs in heart disease [9]. Consistent with the experimentally verified versions, two key regulators miR-1 and miR-21 that were believed as the versatile miRNAs and the most potential target candidates for the treatment of MI and HF passed our strict screening limits. Especially, for miR-1 and miR-21, large quantity of their validated target genes made our result reliable. While false positive protein-protein interactions and wrong miRNA target prediction may be inevitable, our results still illustrated that the therapeutic potential of miRNAs can be distinguished by investigating the inherent variability of miRNA's gene-targeting and are also important for further speculating the biological roles of miRNAs in human heart.

\section{Disclosure Statement}

No declared.

\section{Acknowledgement}

This work was supported by Major Program of National Natural Science Foundation of China (81230081), Research Fund for the Doctoral Program of Higher Education (20112307130004) and National Natural Science Foundation of China (31301095). The funders had no role in study design, data collection and analysis, decision to publish or preparation of the manuscript.

\section{References}

1 Asli NS, Pitulescu ME, Kessel M: MicroRNAs in Organogenesis and Disease. Curr Mol Med 2008;8:698-710.

2 Pan ZW, Lu YJ, Yang BF: MicroRNAs: a novel class of potential therapeutic targets for cardiovascular diseases. Acta Pharmacol Sin 2010;31:1-9.

3 Small EM, Olson EN: Pervasive roles of microRNAs in cardiovascular biology. Nature 2011;469: 336-342.

4 Wang L, Cui Y, Tang M, Hu X, Luo H, Hescheler J, Xi J: Puerarin facilitates T-tubule development of murine embryonic stem cell-derived cardiomyocytes. Cell Physiol Biochem 2014;34:383-92.

5 Li M, Hu X, Zhu J, Zhu C, Zhu S, Liu X, Xu J, Han S, Yu Z: Overexpression of miR-19b impairs cardiac development in zebrafish by targeting ctnnb1. Cell Physiol Biochem 2014;33:1988-2002.

6 Ning B, Qi X, Li Y, Liu H, Zhang F, Qin C: Biventricular pacing cardiac contractility modulation improves cardiac contractile function via upregulating SERCA2 and miR-133 in a rabbit model of congestive heart failure. Cell Physiol Biochem 2014;33:1389-99.

7 Tu Y, Wan L, Bu L, Zhao D, Dong D, Huang T, Cheng Z, Shen B: MicroRNA-22 downregulation by atorvastatin in a mouse model of cardiac hypertrophy: a new mechanism for antihypertrophic intervention. Cell Physiol Biochem 2013;31:997-100

8 Elmén J, Lindow M, Schütz S, Lawrence M, Petri A, Obad S, Lindholm M, Hedtjärn M, Hansen HF, Berger U, Gullans S, Kearney P, Sarnow P, Straarup EM, Kauppinen S: LNA-mediated microRNA silencing in nonhuman primates. Nature 2008;452:896-899.

9 Diez D, Wheelock AM, Goto S, Haeggström JZ, Paulsson-Berne G, Hansson GK, Hedin U, Gabrielsen A, Wheelock CE: The use of network analyses for elucidating mechanisms in cardiovascular disease. Mol Biosyst 2010;6:289-304. 


\section{Cellular Physiology Cell Physiol Biochem 2015;36:1628-1643 \begin{tabular}{l|l} 
DOI: 10.1159/000430324 & (c) 2015 S. Karger AG, Basel
\end{tabular} \\ Xu et al.: Revealing Versatile MicroRNAs in Heart}

10 Pillai RS, Bhattacharyya SN, Filipowicz W: Repression of protein synthesis by miRNAs: how many mechanisms. Trends Cell Biol 2007;17:118-126.

11 Naeem H, Küffner R, Csaba G, Zimmer R: miRSel: automated extraction of associations between microRNAs and genes from the biomedical literature. BMC Bioinformatics 2010;11:135.

12 Van Rooij E, Sutherland LB, Liu N, Williams AH, McAnally J, Gerard RD, Richardson JA, Olson EN: A signature pattern of stress-responsive microRNAs that can evoke cardiac hypertrophy and heart failure. Proc Natl Acad Sci USA 2006;28:18255-18260.

13 Ikeda S, Pu WT: Expression and Function of MicroRNAs in Heart Disease. Curr Drug Targets 2010;11:913925.

14 Naga Prasad SV, Duan ZH, Gupta MK, Surampudi VS, Volinia S, Calin GA, Liu CG, Kotwal A, Moravec CS, Starling RC, Perez DM, Sen S, Wu Q Plow EF, Croce CM, Karnik S: Unique microRNA profile in endstage heart failure indicates alterations in specific cardiovascular signaling networks. J Biol Chem 2009;284:27487-27499.

15 Bostjancic E, Zidar N, Glavac D: MicroRNA microarray expression profiling in human myocardial infarction. Dis Markers 2009;27:255-268.

16 Lloyd-Jones DM, Larson MG, Leip EP, Beiser A, D'Agostino RB, Kannel WB, Murabito JM, Vasan RS, Benjamin EJ, Levy D: Lifetime risk for developing congestive heart failure: the Framingham Heart study. Circulation 2002;106:3068-3072.

17 Bruford EA, Lush MJ, Wright MW, Sneddon TP, Povey S, Birney E: The HGNC Database in 2008: a resource for the human genome. Nucleic Acids Res 2008;36:D445-D448.

18 Shannon P, Markiel A, Ozier O, Baliga NS, Wang JT, Ramage D, Amin N, Schwikowski B, Ideker T: Cytoscape: a software environment for integrated models of biomolecular interaction networks. Genome Res 2003;13:2498-2504.

19 Martin A, Ochagavia ME, Rabasa LC, Miranda J, Fernandez-de-Cossio J, Bringas R: BisoGenet: a new tool for gene network building, visualization and analysis. BMC Bioinformatics 2010;11:91.

20 Yang L, Walker JR, Hogenesch JB, Thomas RS: NetAtlas: a Cytoscape plugin to examine signaling networks based on tissue gene expression. In Silico Biol 2008;8:47-52.

21 Keshava Prasad TS, Goel R, Kandasamy K, Keerthikumar S, Kumar S, Mathivanan S, Telikicherla D, Raju R, Shafreen B, Venugopal A, Balakrishnan L, Marimuthu A, Banerjee S, Somanathan DS, Sebastian A, Rani S, Ray S, Harrys Kishore CJ, Kanth S, Ahmed M, Kashyap MK, Mohmood R, Ramachandra YL, Krishna V, Rahiman BA, Mohan S, Ranganathan P, Ramabadran S, Chaerkady R, Pandey A: Human Protein Reference Database--2009 update. Nucleic Acids Res 2009;37:D767-D772.

22 Yassen A, Fidel R, Sven-Eric S, Thomas L, Mario A: Computing topological parameters of biological networks. Bioinformatics 2008;24:282-284.

23 Naeem H, Küffner R, Csaba G, Zimmer R: miRSel: automated extraction of associations between microRNAs and genes from the biomedical literature. BMC Bioinformatics 2010;11:135.

24 Xiao F, Zuo Z, Cai G, Kang S, Gao X, Li T: miRecords: an integrated resource for microRNA-target interactions. Nucleic Acids Res 2009;37:D105-110.

25 Sam GJ, Harpreet KS, Stijn van D, Anton J: Enright miRBase: tools for microRNA genomics. Nucleic Acids Res 2008;36:154-158.

26 Lall S, Grün D, Krek A, Chen K, Wang YL, Dewey CN, Sood P, Colombo T, Bray N, Macmenamin P, Kao HL, Gunsalus KC, Pachter L, Piano F, Rajewsky N: A genome-wide map of conserved microRNA targets in C. elegans. Curr Biol 2006;16:460-471.

27 Friedman RC, Farh KK, Burge CB, Bartel DP: Most mammalian mRNAs are conserved targets of microRNAs. Genome Res 2009;19:92-105.

28 Zhu W, Yang L, Shan H, Zhang Y, Zhou R, Su Z, Du Z: Clinical Advantage of Propranolol Reveals Key MicroRNAs in Myocardial Infarction. PLoS One 2011;6:e14736.

29 Barabási AL, Oltvai ZN: Network biology: understanding the cell's functional organization. Nat Rev Genet 2009;5:101-113.

30 Anyela C, Francisco A: Linking Gene Expression and Functional Network Data in Human Heart Failure. PLoS ONE 2007;2:e1347.

31 Maslov S, Ispolatov I: Propagation of large concentration changes in reversible protein-binding networks. Proc Natl Acad Sci USA 2007;104:13655-13660. 


\section{Cellular Physiology Cell Physiol Biochem 2015;36:1628-1643 \begin{tabular}{l|l|l}
\hline DOI: 10.1159/000430324 & (C) 2015 S. Karger AG, Basel
\end{tabular} \\ Xu et al.: Revealing Versatile MicroRNAs in Heart}

32 Li F, Li P, Xu W, Peng Y, Bo X, Wang S: PerturbationAnalyzer: a tool for investigating the effects of concentration perturbation on protein interaction networks. Bioinformatics 2010;26:275-277.

33 Da WH, Brad TS, Richard AL: Systematic and integrative analysis of large gene lists using DAVID bioinformatics resources. Nat Protoc 2009;4:44-57.

34 Tusher VG, Tibshirani R, Chu G: Significance analysis of microarrays applied to the ionizing radiation response. Proc Natl Acad Sci U S A 2001;98:5116-5121.

35 Yu W, Wulf A, Liu T, Khoury MJ, Gwinn M: Gene Prospector: An evidence gateway for evaluating potential susceptibility genes and interacting risk factors for human diseases. BMC Bioinformatics 2008;9:528.

36 Reimand J, Arak T, Vilo J: g:Profiler--a web server for functional interpretation of gene lists (2011 update). Nucleic Acids Res 2011;39:W307-W315.

37 Hsu SD, Tseng YT, Shrestha S, Lin YL, Khaleel A, Chou CH, Chu CF, Huang HY, Lin CM, Ho SY, Jian TY, Lin FM, Chang TH, Weng SL, Liao KW, Liao IE, Liu CC, Huang HD: miRTarBase update 2014: an information resource for experimentally validated miRNA-target interactions. Nucleic Acids Res 2014;42:D78-D85.

38 Liu H, Liu W, Liao Y, Cheng L, Liu Q, Ren X, Shi L, Tu X, Wang QK, Guo AY: CADgene: a comprehensive database for coronary artery disease genes. Nucleic Acids Res 2011;39:D991-D996.

39 Finnerty JR, Wang WX, Hébert SS, Wilfred BR, Mao G, Nelson PT: The miR-15/107 group of microRNA genes: evolutionary biology, cellular functions, and roles in human diseases. J Mol Biol 2010;402:491-509.

40 Jazbutyte V, Thum T: MicroRNA-21: from cancer to cardiovascular disease. Curr Drug Targets 2010;11:926935.

41 Baek D, Villén J, Shin C, Camargo FD, Gygi SP, Bartel DP: The impact of microRNAs on protein output. Nature 2008;455:64-71.

42 Arvey A, Larsson E, Sander C, Leslie CS, Marks DS: Target mRNA abundance dilutes microRNA and siRNA activity. Mol Syst Bio 2010;6:363.

43 Kefas B, Godlewski J, Comeau L, Li Y, Abounader R, Hawkinson M, Lee J, Fine H, Chiocca EA, Lawler S, Purow B: microRNA-7 inhibits the epidermal growth factor receptor and the Akt pathway and is downregulated in glioblastoma. Cancer Res 2008;68:3566-3572.

44 Webster RJ, Giles KM, Price KJ, Zhang PM, Mattick JS, Leedman PJ: Regulation of epidermal growth factor receptor signaling in human cancer cells by microRNA-7. J Biol Chem 2009;284:5731-5741.

45 Li X, Cassidy JJ, Reinke CA, Fischboeck S, Carthew RW: A microRNA imparts robustness against environmental fluctuation during development. Cell 2009;137:273-282.

46 Divakaran V, Mann DL: The emerging role of microRNAs in cardiac remodeling and heart failure. Circ Res 2008;103:1072-1083.

47 Eapen Z, Rogers JG: Strategies to attenuate pathological remodeling in heart failure. Curr Opin Cardiol 2009;24:223-229.

48 Larsen JA, Kadish AH, Schwartz JB: Proper use of antiarrhythmic therapy for reduction of mortality after myocardial infarction. Drugs Aging 2000;16:341-350

49 Maslov S, Sneppen K: Specificity and stability in topology of protein networks. Science 2002;296: 910-913.

50 Yang B, Lu Y, Wang Z: Control of cardiac excitability by microRNAs. Cardiovasc Res 2008;79: 571-580.

51 Lu Y, Zhang Y, Shan H, Pan Z, Li X: MicroRNA-1 downregulation by propranolol in a rat model of myocardial infarction: a new mechanism for ischaemic cardioprotection. Cardiovasc Res 2009;84:434-441.

52 Sayed D, Hong C, Chen IY, Lypowy J, Abdellatif M: MicroRNAs play an essential role in the development of cardiac hypertrophy. Circ Res 2007;100:416-424.

53 Bonci D,:MicroRNA-21 as therapeutic target in cancer and cardiovascular disease. Recent Pat Cardiovasc Drug Discov 2010;5:156-161.

54 Ghaemmaghami S, Huh WK, Bower K, Howson RW, Belle A, Dephoure N, O'Shea EK, Weissman JS: Global analysis of protein expression in yeast. Nature 2003;425:737-741.

55 Selbach M, Schwanhäusser B, Thierfelder N, Fang Z, Khanin R, Rajewsky N: Widespread changes in protein synthesis induced by microRNAs. Nature 2008;455:58-63.

56 Cordes KR, Sheehy NT, White MP, Berry EC, Morton SU, Muth AN, Lee TH, Miano JM, Ivey KN, Srivastava D: miR-145 and miR-143 regulate smooth muscle cell fate and plasticity. Nature 2009;460:705-710.

57 Tang WH, Francis GS: The year in heart failure. J Am Coll Cardiol 2010;55:688-696. 